
\title{
28 Research Square \\ Evaluation of the Effects of Chitosan on Methotrexate-Induced Oral Mucositis in Rats
}

\section{Kani Bilginaylar ( $\nabla$ kani_blgnylr@hotmail.com )}

Final International University: Uluslarasi Final Universitesi https://orcid.org/0000-0003-3893-2471

\section{Asli Aykac}

Near East University: Yakin Dogu Universitesi

\section{Serkan Sayiner}

Near East University: Yakin Dogu Universitesi

\section{Hanife Özkayalar}

Near East University: Yakin Dogu Universitesi

Ahmet Özer Şehirli

Near East University: Yakin Dogu Universitesi

\section{Research Article}

Keywords: Apoptosis, chitosan, cytokines, matrix metalloproteinases, oral mucositis

Posted Date: November 29th, 2021

DOI: https://doi.org/10.21203/rs.3.rs-1089228/v1

License: (c) (i) This work is licensed under a Creative Commons Attribution 4.0 International License. Read Full License 


\section{Abstract}

I. Background: Methotrexate (MTX), a chemotherapeutic agent, is known to cause oral mucositis. Chitosan has been shown to have a protective effect in inflammatory animal models. This research aimed to examine the protective effect of chitosan against oral mucositis caused by MTX.

II. Methods and Results: Wistar albino rats were randomly divided into three groups, 8 in each group as follow: Control (saline via oral gavage for 5 days), MTX (60 mg/kg single dose MTX intraperitoneally on 1 st day and for following 4 days saline via oral gavage), and MTX+Chitosan(1st day single dose 60 $\mathrm{mg} / \mathrm{kg}$ MTX intraperitoneally and followed with $200 \mathrm{mg} / \mathrm{kg}$ Chitosan via oral gavage for 4 days). After 24 hours of the last dose, animals were euthanised. Blood, tongue, buccal and palatal mucosa tissues were collected. Serum interleukin 1-beta (IL1- $\beta$ ), tumour necrosis factor-alpha (TNF-a), matrix metalloproteinase (MMP-1, and MMP-2) activities, and tissue bcl-2/bax ratio and the expression of caspase-3 (casp-3), and casp-9 were detected. The tissues were also examined histologically. Serum TNFa, IL1- $\beta$, MMP-1 and MMP-2 activities and tissue casp-3 and casp-9 activities significantly increased but the bcl-2/bax ratio significantly decreased in the MTX group compared to the control group. Histologically, diffuse inflammatory cells were observed in MTX group. However, In the MTX + Chitosan group, all parameters approached the values of control group.

III. Conclusion: Chitosan has been found to have a protective effect against oral mucosal damage caused by MTX. Thus, it may be a candidate agent against MTX induced oral mucositis.

\section{Introduction}

In cancer treatment, chemotherapy drugs play central roles, which have significant side effects on proliferative tissues. Radiotherapy combined with chemotherapy is another factor affecting dividing cells [1]. These patients who receive dual therapy are among the high-risk groups in terms of toxicity $[2,3]$. One of the most common and devastating side effects observed with these treatments is oral mucositis. Oral mucositis leads to atrophy and ulceration with potentially serious complications in oral mucosa [4-6]. Severe oral mucositis, which occurs as a treatment complication, causes an interruption in anticancer treatments and significantly decreases patients' quality of life. Therefore, it is crucial to prevent or promptly treat oral mucositis symptoms $[7,8]$. Various anti-inflammatory drugs and mouthwashes are using to treat oral mucositis, but it has reported that there is not enough therapeutic effect $[9,10]$. Studies on oral mucositis have focused on the mechanisms involved in the generation of toxicity and highlighted apoptosis genes' key roles [11-14]. The ultimate purposes of mucositis physiopathology studies were to reduce or even prevent mucositis formation and determine the effect of radical therapy on target mechanisms [14-16].

In this study, Methotrexate (MTX) was used to induce oral mucositis. It is an antiproliferative folic acid analogue, an inhibitor of dihydrofolate reductase and an agent used in various chronic inflammatory diseases and cancer treatment [17]. In inflammatory conditions, low doses use, whereas high doses in 
malignancies. High MTX doses cause serious side effects. MTX damages the oral tissues, primarily by destroying the mucous layer. MTX destroys rapidly dividing cells in the body, such as the gastrointestinal epithelium, including the buccal epithelium [6]. The use of MTX mainly causes oral mucositis with a rate of $40 \%$ in cancer chemotherapy. Pro-inflammatory cytokines and reactive oxygen species play an essential role in forming mucositis [18-21].

Apoptosis plays an essential role in many physiological processes, including pathologies in the mucosa [22]. Although the distinctive feature of many degenerative disorders is excessive apoptosis, reduced or absent apoptosis observe in some proliferative diseases [23]. It has known that MTX generally damages the proliferation of basal epithelial cells in the oral mucosa and cause oral mucositis in cancer patients [24]. Matrix metalloproteinases (MMPs) act as modulators in both regulating the homeostasis of the extracellular matrix and the response to inflammation and tissue damage during mucositis development. They have essential enzyme roles in mucosal physiology. Previous studies have reported that these enzymes participate in the ulcerative phase of oral mucositis by disrupting normal cell kinetics. They cause mucosal pathologies by mediating apoptosis $[25,26]$.

Chitosan is a degradable and non-toxic biopolymer obtained by deacetylation of chitin and used to treat oral mucositis. It is superior to other biopolymers due to its properties. Its antioxidant properties have reported in previous studies. Also, it has been shown in the literature that chitosan prevents tissue damage with its antioxidant and anti-inflammatory properties [27-29]. Moreover, it is non-toxic and biodegradable that makes chitosan superior to other biopolymers [29,30]. Because of these significant effects of chitosan, we planned to investigate the protective effects of Chitosan against to MTX induced oral mucositis in the presented study.

Rats, medium-sized, long-tailed rodents, are ordinarily utilized in animal experiments of tissue injuries that resulted from inflammation as they are very similar to human beings genetically [31, 32]. In this study, the response of the methotrexate-induced mucositis to chitosan treatment was investigated using a rat model. This study aimed to examine and demonstrate the alterations in systemic and tissue levels caused by chitosan treatment and its healing effects biochemically, immunohistochemically and histopathologically.

\section{Material And Method 2.1. Animals}

The animals were obtained from the Near East University Animal Experiments Unit. The rats were maintained under a $12: 12 \mathrm{~h}$ dark/light cycle from the start at a temperature of $22 \pm 2^{\circ} \mathrm{C}$ in the humiditycontrolled room (50 $\pm 5 \%$ ) under ad libitum feeding without any restriction on their standard rat chow and water. The rats were housed in each plexiglass cage (4 rats/cage, $60 \times 40 \times 40 \mathrm{~cm}$ ). Ethical approval of research was obtained from the Near East University Local Animal Experiments Ethics Committee on 17/04/2020 / no: 2020/111. 


\subsection{Experimental design and induction of experimental oral mucositis}

The study was randomized, controlled, single-centre, single-blind (the study analyst was blind to the medications administered to the rats), and gender selected homogeneously (i.e., four males and four female rats for each group). In the study, 24 Wistar Albino rats of both sexes used, weighing $200-250 \mathrm{~g}$, eight rats in each group randomly divided into three groups. 1. group (control, $n=8$ ): no procedure applied to the animals, only saline solution was administered by oral gavage for 5 days. 2 . group (MTX, $n=8)$ : MTX was administered single dose $(60 \mathrm{mg} / \mathrm{kg})$ intraperitoneally on 1st day and for following 4 days, saline solution was administered[20]. 3. group (MTX + Chitosan, $\mathrm{n}=8): 1$ st day single dose $(60 \mathrm{mg} / \mathrm{kg})$ MTX was administered intraperitoneally and followed with Chitosan $(200 \mathrm{mg} / \mathrm{kg})$ administration by oral gavage for 4 days [27]. All animals observed for 5 days.

Twenty-four hours after the last injection, all rats were euthanized by decapitation. After killing all animals, blood samples were collected into serum separator tubes and delivered to Diagnostic Laboratory of Animal Hospital, Near East University. Sera were separated at $1500 \mathrm{~g} \times 10$ minutes and stored at $-80^{\circ} \mathrm{C}$ until analyses. Tongue, buccal and palatal mucosa tissues were extracted. Interleukin 1-beta (IL1- $\beta$ ), tumour necrosis factor-alpha (TNF-a), matrix metalloproteinase (MMP-1, and MMP-2) activities were analyzed spectrophotometrically (Mindray, BS120 automated biochemistry analyzer) in the serum for biochemical analysis. The bcl-2/bax ratio, the expression of caspase-3 (casp-3), and casp-9 were analyzed in all tissues by the western blotting method and examined histologically.

\subsection{Biochemical Analysis}

TNF-a, IL-1 $\beta$, MMP-1 and MMP-2 were measured on serum samples following the manufacturer's instructions and guidelines using commercially available rat specific enzyme- linked immunosorbent assay (ELISA) kits (ELR-TNF-a-2; ELR-IL1ß -2; ELR-MMP1-2; ELR-MMP2-2, RayBiotech Inc., GA 30092, USA). The washing steps were performed using an automated microtiter washer (MW-12A Microplate washer, Mindray, Shenzhen, China). The samples' optical density was obtained using a microtiter plate reader (MR-96A Microplate reader, Mindray), and results were calculated according to instructions.

\subsection{Western blotting}

After killing the rat, all tissues (tongue, palatal and buccal mucosa) were dissected, frozen, and stored at $-80^{\circ} \mathrm{C}$. Extracted tissues of four animals from each group homogenized and $10 \mathrm{~min}$. centrifuged at 2000 G, lastly incubated with $0.1 \mathrm{mM}$ EDTA, $0.5 \mathrm{mM} \mathrm{DTT}$, \% glycerol, $0.0 \%$ Triton X-100, protease inhibitors and $10 \mathrm{mM}$ Tris- $\mathrm{HCl}$ for 1 hour. The amount of protein in each tissue determined by Lowry method [33]. $100 \mu \mathrm{g}$ protein was used for each tissue to prepare samples and loaded on gel electrophoresis (12\% SDS-PAGE gel). Then samples were put into the nitrocellulose membranes (Schleicher and Schuell, $0.45 \mathrm{~m}$, Germany) for 75 minutes at $80 \mathrm{~V}$. The incubation of all membranes done using Alkaline phosphataseconjugated rabbit monoclonal anti-goat IgG as the secondary antibodies for 1 hour. After, 16 hours' incubation with primary antibodies [bax (1:100; sc-20067), bcl-2 (1:200; sc-7382), casp-3 (1:200; sc- 
56053), and casp-9 (1:200; sc-56076) at $+4{ }^{\circ} \mathrm{C}$, $\beta$-actin (1:100; sc-130657) was used for standardization in all membranes. Finally, the Bio-Rad Molecular Analyst Software used for the densitometric analysis of the membranes (www.totallab.com, Free). Molecular masses for bax, bcl-2, casp-3, casp-9 and $\beta$-actin are $23,26,35,20$, and $43 \mathrm{kDa}$, respectively.

\subsection{Histological examination and light microscopic analysis}

Hematoxylin and Eosin (H\&E) staining Paraffin wax blocks of the fixed tongue, palatal and buccal mucosa tissues were sliced as $4 \mu \mathrm{m}$ sections by using a rotary microtome and H\&E used for histological staining. Olympus Microscope (BH-6, Shinjuku, Tokyo, Japan) used for histological inspection of H\&E stained sections. Micrographs set at $200 \times$ magnification (Olympus Altra 20). The epithelial thickness of the oral mucosa was measured using xxx software. An average of 15 to 20 measurements was recorded between the surface and basal layers of the epithelium's chosen piece.

\subsection{Statistical analysis}

Statistical analyses were performed using GraphPad Prism 3.0 (GraphPad Software, San Diego, CA, USA). Quantitative data presented as mean \pm standard error of mean (SEM) for eight animals in each group. To compare differences between more than two independent variables, one-way analysis of variance (ANOVA) was employed and followed for determining the differences between multiple groups; Tukey's multiple comparison tests were used. A value of $p \leq 0.05$ was taken to indicate statistical significance.

\section{Results}

\subsection{Serum Levels of TNF- $a$, IL-1 $\beta$, MMP-1 and MMP-2.}

Serum levels of pro-inflammatory cytokines (TNF- $\alpha$, IL-1 $\beta$ ) and matrix metalloproteinases, (MMP-1, and MMP-2) were significantly higher in the group 1 (MTX-induced oral mucositis) than the control group in all tissues $(p<0.001)$. On the other hand, it determined that the increase in group 2 (MTX) almost returned to control values with Chitosan treatment (group 3, MTX + Chitosan), ( $<<0.05-0.001$; Fig. 1).

\subsection{Western blotting analyzes}

Western blotting experiments performed to investigate the effect of chitosan treatment on the bax, bcl-2, casp-3, and casp-9 protein levels in the tongue, palatal and buccal mucosa tissues of MTX-induced oral mucositis of rats ( $n=8$ for each group). The membranes and representative western blottings of the oral tissues were illustrated in figure 2 (all membranes normalized by using a $\beta$-actin antibody).

The expression level of bax was statistically significantly increased in the MTX group than the control group in all examined tissues. The expression level of bax was substantially higher in the MTX group than the chitosan-treated group. Conversely, in the MTX group, the levels of bcl-2 were significantly lesser 
compared to the control and MTX + Chitosan groups. Moreover, expression levels were similar in all tissues between the control and MTX + Chitosan groups. In the MTX group, the bcl-2/bax ratio was significantly lower compared to the control $(p<0.0001$, for all tissues) and the MTX + Chitosan groups $(p<0.01-0.001$; Fig. 3a-3c).

When the casp- 9 expression analyzed in all regions determined that the casp- 9 expression levels of MTX group rats increased compared to the control group ( $p<0.05$, for all tissues), however, in group 3 , the chitosan treatment caused the casp- 9 expression levels to increase compared to the MTX group in all tissues ( $p<0.05$; Fig. 3d-3f).

The casp-3 expressions were markedly elevated in the MTX group in all regions compared to the control group ( $p<0.05-0.01)$. MTX + Chitosan significantly decreased casp-3 expression levels compared to the MTX group in all regions ( $p<0.01-0.001$; Fig. 3g-3i).

\subsection{Histological Analyses}

Tongue tissue: Inflammatory cells were not observed in submucosa (control group), moderate inflammatory cells were found in submucosa (MTX group) and rare inflammatory cell was found in submucosa (MTX + Chitosan group), (Fig. 4a, 4b, 4c, respectively).

Palatal tissue: Inflammatory cells were not detected in submucosa (control group), inflammatory cells were found in submucosa (MTX group), rare inflammatory cell was found in submucosa (MTX + Chitosan group), (Fig. 5a, 5b, 5c, respectively).

Buccal tissue: Inflammatory cells were not observed in submucosa (control group), moderate inflammatory cells were seen in submucosa and lymphoid aggregate was found in muscular layer (MTX group), rare inflammatory cell was observed in submucosa (MTX + Chitosan group), (Fig. 6a, 6b, 6c, respectively).

\section{Discussion}

Chemotherapy and radiotherapy cause damage to the mucosal surface, resulting in difficulty eating, drinking, and swallowing [34]. In this study, the effect of chitosan on oral mucositis caused by MTX evaluated by taking the tongue, palatal and buccal tissues of rats. Inflammation assessed by examining the cytokine (IL-1 $\beta$ and TNF-a) expression and MMPs (MMP-1, MMP-2) in blood and the levels of bcl-2 / bax ratio, caspase-3, caspase- 9 in the tissues at the end of the experiment. Furthermore, structural damage was evaluated by histological examinations.

In this study, we found that blood IL-1 $\beta$ and TNF-a levels increased with MTX administration to the rats, and these cytokine levels decreased to control levels with chitosan administration. This result is compatible with the literature and evaluated due to the development of mucositis with chemotherapy [35]. It has shown that with the application of MTX, the increase in IL-1 $\beta$ and TNF-a levels in the blood plays a role in the mechanism of damage in different tissues [36]. It has also shown that chitosan 
reduces increased levels of IL-1 $\beta$ and TNF- $\alpha$ in inflammation models in rats [37]. Both our results and the findings of the literature suggest that chitosan has anti-inflammatory properties and thus may be effective in oral mucositis damage.

MMPs regulate apoptotic and anti-apoptotic actions in cellular death in an intriguing way [38]. For example, MMP-2 separates stromal cell-derived factor-a and cause neural death of the cell. Further, the apoptosis of basal keratinocytes of the oral mucosa is associated with MMPs. Since it thought to upregulate the factors involved in apoptosis [22]. Previous in vivo studies showed that the lack of epithelial cells in the underlying basement membrane and subsequently lack of the source of MMPs, upregulation of apoptosis-related genes and finally, death of cell documented [39]

Moreover, in cancer studies, the anti-apoptotic activity of MMPs have also determined by stimulating tumour invasion and metastasis. Therefore, it secures apoptosis of the invading tumour cells [40]. MMPs changes the micro-environment and alter cellular behaviour by affecting ECM molecules, including morphogenesis and cell proliferation [41]. MMPs and their inhibitors also occur in such oral mucosal disorders as oral lichen planus and aphthous ulceration [42, 43]. Besides, some gastrointestinal diseases may cause symptoms in the oral mucosa; therefore, it is logical to speculate common pathophysiology in both mucosas. Mutual pathophysiological processes of autoimmune diseases, such as pemphigus, pemphigoid, and systemic lupus erythematosus, should be considered while investigating some inflammatory mediated skin diseases which affect the oral mucosa [44]. Additionally, in Stevens-Johnson syndrome, hypersensitivity reaction lesions occur, and toxic epidermal necrolysis may widely participate in the oral mucosa [45].

MMPs are related to the pathophysiology of these conditions. In our study, it has shown that serum MMP1 and MMP-2 levels increased with methotrexate administration. These results are consistent with the literature. MMP-1 and MMP-2 levels approached control values with Chitosan application. The effects of chitosan on MMP-1 and MMP-2 expressions have studied in various models. In these models, chitosan has a protective effect with its anti-inflammatory effects. However, the impact of chitosan on oral mucositis has not studied before. With this study we have done, chitosan will prevent oral mucositis that may occur during MTX treatment.

Mitochondria play a significant role in apoptosis. MTX is known to induce apoptosis along with mitochondrial dysfunction. There is a balance between anti-apoptotic and proapoptotic genes [46]. While Bax causes the activation of apoptosis, the bcl-2 protein exerts an anti-apoptotic effect by preventing Bax activation. Caspases act as the primary applicators of apoptosis. The apoptosis in methotrexate-induced oral mucositis and determination of apoptosis by using DNA fragmentation observed in only one study[19].

DNA fragmentation is an indicator of apoptosis and its necrosis and is not a suitable marker for apoptosis. Our study demonstrated the importance of apoptosis in mucositis by evaluating the bcl-2 / bax ratio and caspase activation. It has shown in the literature that chitosan used in this study prevents 
apoptosis in addition to its anti-inflammatory effect. These results are consistent with the results of the present research.

In this study, increased inflammatory cells were detected in all tissues in the methotrexate group while the researchers evaluated histopathological examinations. A structural improvement observed with chitosan. Studies have shown that damage occurs to the mucosa due to chemotherapeutic agents inducing cytokine levels and the apoptotic process $[35,47]$. Our study observed that chitosan contributes to tissue healing thanks to its anti-inflammatory properties, following the literature.

As a result, participating researchers in this study found that chitosan exerts a protective effect by suppressing the cytokine expression and apoptotic processes in oral mucositis formed in fornix, tongue, and palate tissues by methotrexate administration. The use of this non-cytotoxic agent together with methotrexate is vital in terms of reducing its toxic effects.

\section{Declarations}

\section{Funding}

The authors declare that no funds, grants, or other support were received during the preparation of this manuscript.

\section{Competing Interests}

The authors have no relevant financial or non-financial interests to disclose.

\section{Author Contributions}

All authors contributed to the study conception and design. Material preparation, data collection and analysis were performed by Kani Bilginaylar, Asli Aykac, Serkan Sayiner, Hanife Ozkayalar and Ahmet Ozer Şehirli. The first draft of the manuscript was written by Kani Bilginaylar and all authors commented on previous versions of the manuscript. All authors read and approved the final manuscript.

\section{Ethics approval}

This study was performed in line with the principles of the Declaration of Helsinki. Approval was granted by the Ethics Committee of the Near East University Local Animal Experiments Ethics Committee on 17/04/2020 / no: 2020/111.

\section{References}

1. DJ H (2006) Cancer treatment-induced mucositis pain: strategies for assessment and management. Therapeutics and clinical risk management 2:251-258. https://doi.org/10.2147/TCRM.2006.2.3.251 
2. S A-A, JA Z, A B, et al (2015) Oral Mucositis Induced By Anticancer Therapies. Current oral health reports 2:202-211. https://doi.org/10.1007/S40496-015-0069-4

3. M C, G A, P E, et al (2017) New Frontiers in the Pathobiology and Treatment of Cancer RegimenRelated Mucosal Injury. Frontiers in pharmacology 8:. https://doi.org/10.3389/FPHAR.2017.00354

4. MM C, LM B (2005) Current trends in managing oral mucositis. Clinical journal of oncology nursing 9:584-592. https://doi.org/10.1188/05.CJON.584-592

5. Munaretto JC, Ponzoni D, Sabbagh-Haddad A, Puricelli E (2011) Preliminary histological analysis of methotrexate-induced oral mucositis: experimental study in mice. Revista da Faculdade de Odontologia - UPF 16:144-148. https://doi.org/10.5335/RFO.V1612.2118

6. Alrifai A, Kamal A (2019) PROTECTIVE ROLE OF HONEY ON THE DORSAL SURFACE OF THE TONGUE OF CHEMOTHERAPY TREATED ALBINO RATS (Immunohistochemical Study) The Effect of Green Tea Extract on Submandibular Salivary Gland of Methotrexate Treated Albino Rats: Immunohistochemical Study View project Protective Role Of Honey on The Dorsal Surface of The Tongue of Methotrexate Treated Rats (Histological and Immunohistochemical Study) View project

7. F B, JE T-S, RF G, PJ P (2005) A critical review of biomarkers used for monitoring human exposure to lead: advantages, limitations, and future needs. Environmental health perspectives 113:1669-1674. https://doi.org/10.1289/EHP.7917

8. TA K, SS M (2011) Modulation of lead biohazards using a combination of epicatechin and lycopene in rats. Human \& experimental toxicology 30:1674-1681. https://doi.org/10.1177/0960327110396536

9. N M, Y E, G N, E K (2009) Protective Effect of Caffeic Acid Phenethyl Ester Against Lead Acetateinduced Hepatotoxicity in Mice. KAFKAS ÜNIVERSITESI VETERINER FAKÜLTESI DERGisi 17:. https://doi.org/10.9775/KVFD.2010.2717

10. $Z$ W, Y Y, X Y, et al (2016) Protective effects of chitosan and its water-soluble derivatives against leadinduced oxidative stress in mice. International journal of biological macromolecules 83:442-449. https://doi.org/10.1016/J.IJBIOMAC.2015.10.017

11. Yeoh ASJ, Gibson RJ, Yeoh EEK, et al (2007) A novel animal model to investigate fractionated radiotherapy-induced alimentary mucositis: The role of apoptosis, p53, nuclear factor-kB, COX-1, and COX-2. Molecular Cancer Therapeutics 6:2319-2327. https://doi.org/10.1158/1535-7163.MCT-070113

12. AS Y, JM B, RJ G, DM K (2005) Nuclear factor kappaB (NFkappaB) and cyclooxygenase-2 (Cox-2) expression in the irradiated colorectum is associated with subsequent histopathological changes. International journal of radiation oncology, biology, physics 63:1295-1303. https://doi.org/10.1016/J.IJROBP.2005.04.041

13. JM B, RJ G, A T, et al (2007) Gene expression analysis of multiple gastrointestinal regions reveals activation of common cell regulatory pathways following cytotoxic chemotherapy. International journal of cancer 121:1847-1856. https://doi.org/10.1002/IJC.22895 
14. N A-D, ST S, JM B, et al (2013) Emerging evidence on the pathobiology of mucositis. Supportive care in cancer: official journal of the Multinational Association of Supportive Care in Cancer 21:20752083. https://doi.org/10.1007/S00520-013-1810-Y

15. Bowen J, Al-Dasooqi N, Bossi P, et al (2019) The pathogenesis of mucositis: updated perspectives and emerging targets. Supportive Care in Cancer 27:4023-4033. https://doi.org/10.1007/S00520019-04893-Z

16. AR A-A, RJ G, DM K, RM L (2013) Matrix metalloproteinases: do they play a role in mucosal pathology of the oral cavity? Oral diseases 19:347-359. https://doi.org/10.1111/ODI.12023

17. PT R, Z Z, L M, et al (2002) Interaction of dihydrofolate reductase with methotrexate: ensemble and single-molecule kinetics. Proceedings of the National Academy of Sciences of the United States of America 99:13481-13486. https://doi.org/10.1073/PNAS.172501499

18. DE P, RV L (2010) Oral mucositis: the new paradigms. Current opinion in oncology 22:318-322. https://doi.org/10.1097/CCO.0B013E32833A9FAB

19. AA A, MA S, NM E-S (2017) a-Lipoic acid ameliorates oral mucositis and oxidative stress induced by methotrexate in rats. Histological and immunohistochemical study. Life sciences 171:51-59. https://doi.org/10.1016/J.LFS.2017.01.001

20. L K, P Y, P B, et al (2019) Effect of local bee honey on dihydrofolate reductase enzyme inhibitorinduced mucositis: A histological study on albino wistar rats. Indian journal of dental research: official publication of Indian Society for Dental Research 30:708-715. https://doi.org/10.4103/IJDR.IJDR_689_17

21. F O, AV K, EM A, et al (2020) Effects of anakinra on the small intestine mucositis induced by methotrexate in rats. Experimental animals 69:144-152. https://doi.org/10.1538/EXPANIM.19-0057

22. Kim SG, Chae CH, Cho BO, et al (2006) Apoptosis of oral epithelial cells in oral lichen planus caused by upregulation of BMP-4. Journal of oral pathology \& medicine: official publication of the International Association of Oral Pathologists and the American Academy of Oral Pathology 35:3745. https://doi.org/10.1111/J.1600-0714.2005.00373.X

23. Mannello F, Luchetti F, Falcieri E, Papa S (2005) Multiple roles of matrix metalloproteinases during apoptosis. Apoptosis: an international journal on programmed cell death 10:19-24. https://doi.org/10.1007/S10495-005-6058-7

24. van der Beek JN, Oosterom N, Pieters R, et al (2019) The effect of leucovorin rescue therapy on methotrexate-induced oral mucositis in the treatment of paediatric ALL: A systematic review. Critical reviews in oncology/hematology 142:1-8. https://doi.org/10.1016/J.CRITREVONC.2019.07.003

25. Al-Dasooqi N, Sonis ST, Bowen JM, et al (2013) Emerging evidence on the pathobiology of mucositis. Supportive Care in Cancer 21:2075-2083. https://doi.org/10.1007/s00520-013-1810-y

26. Al-Azri AR, Gibson RJ, Keefe DMK, Logan RM (2013) Matrix metalloproteinases: do they play a role in mucosal pathology of the oral cavity? Oral diseases 19:347-359. https://doi.org/10.1111/ODI.12023 
27. Toz H, Değer Y (2018) The Effect of Chitosan on the Erythrocyte Antioxidant Potential of Lead Toxicity-Induced Rats. Biological Trace Element Research 184:114-118.

https://doi.org/10.1007/S12011-017-1164-2

28. Smith DL, Harris AD, Johnson JA, et al (2002) Animal antibiotic use has an early but important impact on the emergence of antibiotic resistance in human commensal bacteria. Proceedings of the National Academy of Sciences of the United States of America 99:6434-6439. https://doi.org/10.1073/PNAS.082188899

29. Wu KY, Wu M, Fu ML, et al (2006) A novel chitosan CpG nanoparticle regulates cellular and humoral immunity of mice. Biomedical and Environmental Sciences 19:87-95

30. Şenel S, Kremer MJ, Kaş S, et al (2000) Enhancing effect of chitosan on peptide drug delivery across buccal mucosa. Biomaterials 21:2067-2071. https://doi.org/10.1016/S0142-9612(00)00134-4

31. Patel A, Rajesh S, Chandrashekhar VM, et al (2013) A rat model against chemotherapy plus radiationinduced oral mucositis. Saudi pharmaceutical journal: SPJ : the official publication of the Saudi Pharmaceutical Society 21:399-403. https://doi.org/10.1016/J.JSPS.2012.11.003

32. Sehirli A, Aksoy U, Kermeoglu F, et al (2019) Protective effect of alpha-lipoic acid against apical periodontitis-induced cardiac injury in rats. European journal of oral sciences 127:333-339. https://doi.org/10.1111/EOS.12618

33. LOWRY OH, ROSEBROUGH NJ, FARR AL, RANDALL RJ (1951) Protein measurement with the Folin phenol reagent. The Journal of biological chemistry 193:265-275. https://doi.org/10.1016/s00219258(19)52451-6

34. Vissink A, Jansma J, Spijkervet FKL, et al (2003) Oral sequelae of head and neck radiotherapy. Critical Reviews in Oral Biology and Medicine 14:199-212. https://doi.org/10.1177/154411130301400305

35. Çakir T, Polat C, Baştürk A, et al (2015) The effect of alpha lipoic acid on rat kidneys in methotrexate induced oxidative injury. European Review for Medical and Pharmacological Sciences 19:2132-2139

36. Kuduban O, Mazlumoglu MR, Kuduban SD, et al (2016) The effect of hippophae rhamnoides extract on oral mucositis induced in rats with methotrexate. Journal of applied oral science: revista FOB 24:423-430. https://doi.org/10.1590/1678-775720160139

37. Jhundoo HD, Siefen T, Liang A, et al (2020) Anti-Inflammatory Activity of Chitosan and 5-Amino Salicylic Acid Combinations in Experimental Colitis. Pharmaceutics 12:1-16. https://doi.org/10.3390/PHARMACEUTICS12111038

38. Egeblad M, Werb Z (2002) New functions for the matrix metalloproteinases in cancer progression. Nature reviews Cancer 2:161-174. https://doi.org/10.1038/NRC745

39. Frisch SM, Francis H (1994) Disruption of epithelial cell-matrix interactions induces apoptosis. Journal of Cell Biology 124:619-626. https://doi.org/10.1083/jcb.124.4.619

40. Bergers G, Coussens LM (2000) Extrinsic regulators of epithelial tumor progression: Metalloproteinases. Current Opinion in Genetics and Development 10:120-127. https://doi.org/10.1016/S0959-437X(99)00043-X 
41. Vu TH, Werb Z (2000) Matrix metalloproteinases: Effectors of development and normal physiology. Genes and Development 14:2123-2133. https://doi.org/10.1101/gad.815400

42. Häyrinen-Immonen R, Sorsa T, Nordström D, et al (1993) Collagenase and stromelysin in recurrent aphthous ulcers (RAU). International Journal of Oral and Maxillofacial Surgery 22:46-49. https://doi.org/10.1016/S0901-5027(05)80357-1

43. Mazzarella N, Femiano F, Gombos F, et al (2006) Matrix metalloproteinase gene expression in oral lichen planus: Erosive vs. reticular forms. Journal of the European Academy of Dermatology and Venereology 20:953-957. https://doi.org/10.1111/j.1468-3083.2006.01693.x

44. Said S, Golitz L (2011) Vesiculobullous eruptions of the oral cavity. Otolaryngologic clinics of North America 44:133-160. https://doi.org/10.1016/J.0TC.2010.09.005

45. Worswick S, Cotliar J (2011) Stevens-Johnson syndrome and toxic epidermal necrolysis: a review of treatment options. Dermatologic therapy 24:207-218. https://doi.org/10.1111/J.15298019.2011.01396.X

46. Abo-Haded HM, Elkablawy MA, Al-Johani Z, et al (2017) Hepatoprotective effect of sitagliptin against methotrexate induced liver toxicity. PloS one 12:. https://doi.org/10.1371/JOURNAL.PONE.0174295

47. Helal MG, Said E (2020) Tranilast attenuates methotrexate-induced renal and hepatic toxicities: Role of apoptosis-induced tissue proliferation. Journal of biochemical and molecular toxicology 34:. https://doi.org/10.1002/JBT.22466

\section{Figures}


a)

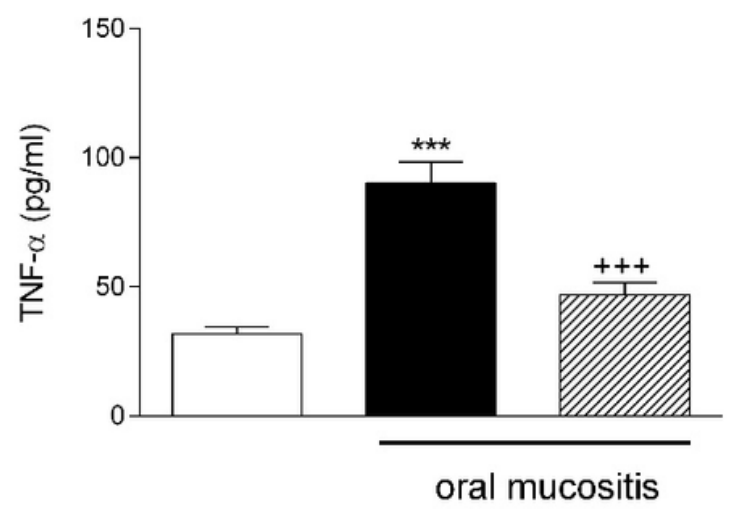

c)

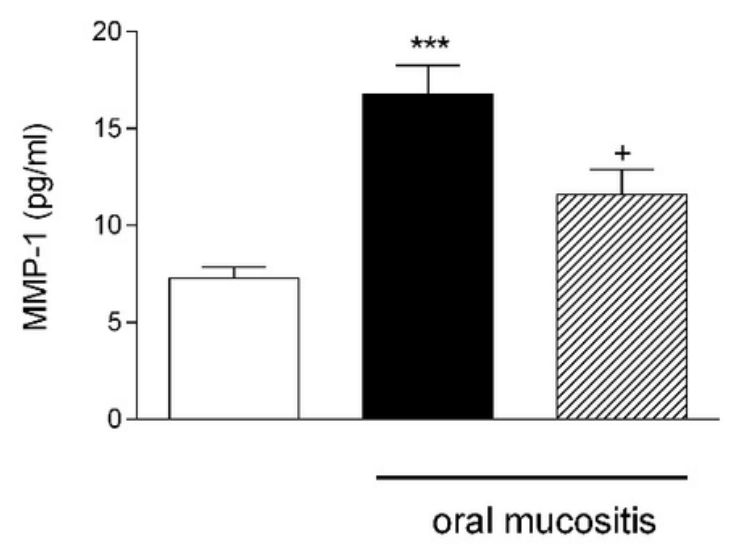

b)

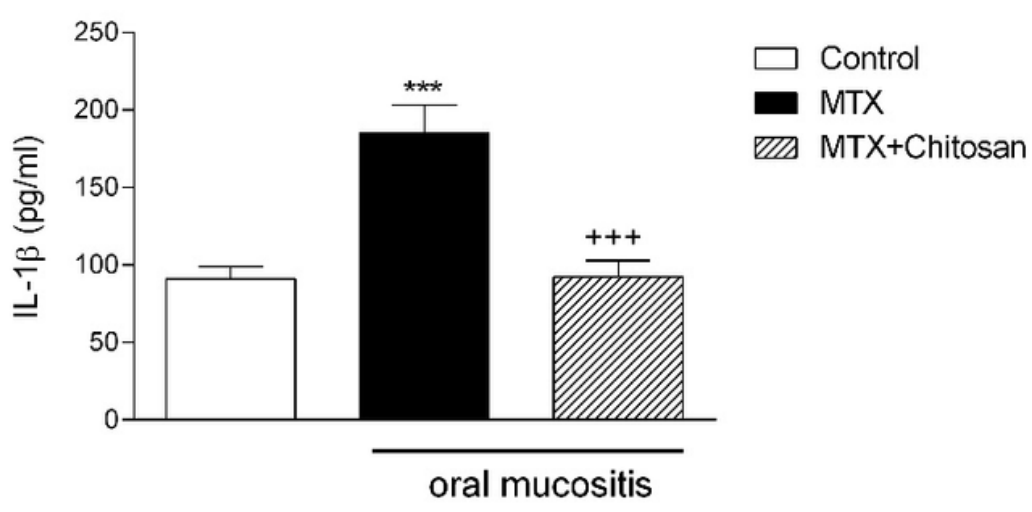

d)

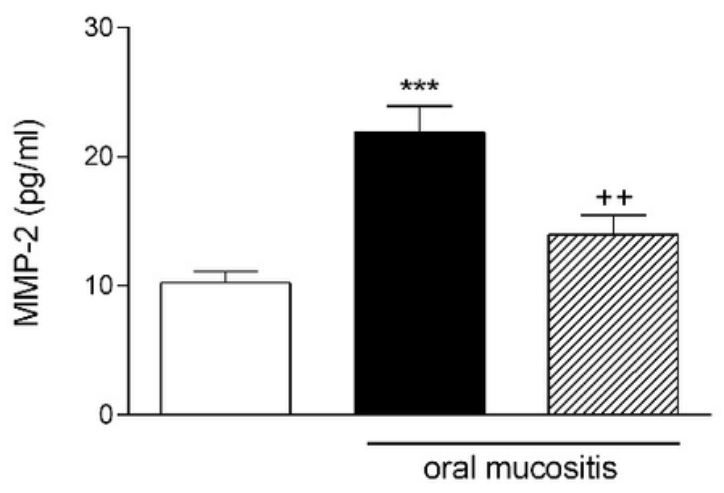

\section{Figure 1}

The levels of serum a) TNF-a, b) IL-1 $\beta$ c) MMP-1 and d) MMP-2 of tongue, palatal, and buccal tissues in the control, MTX, and MTX + Chitosan groups. ${ }^{* \star *} \mathrm{p}<0.001$ Comparisons according to control group, +++ $p<0.001$ Comparisons according to MTX group. 


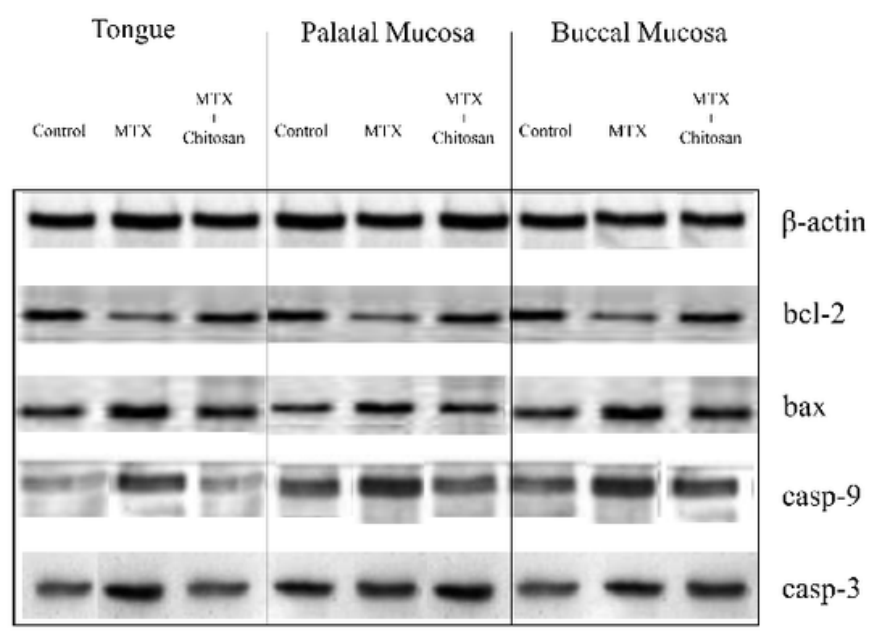

\section{Figure 2}

The representative images of nitrocellulose membranes obtained from immunoblotting experiments of the tongue, palatal, and buccal tissues in control, MTX, and chitosan-treated groups protein expressions of bax, bcl 2, 囚-actin, casp-3, and casp-9. 

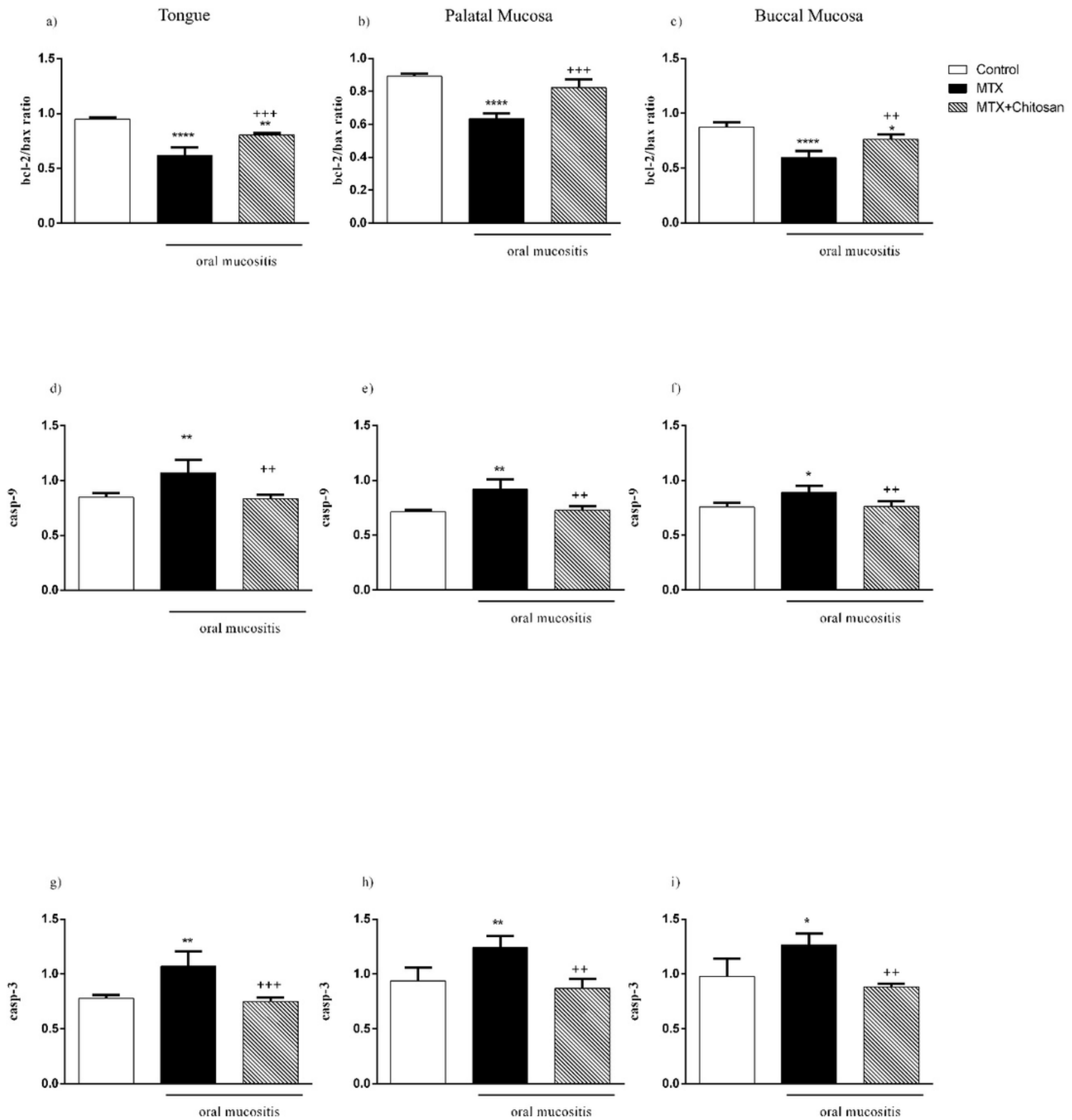

h)

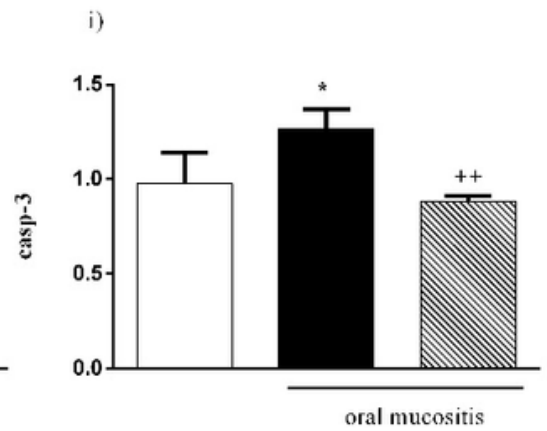

Figure 3

The expression levels of bcl-2 / bax ratio, casp-3 and casp-9 of tongue, palatal, and buccal tissues in the control, MTX, and MTX + Chitosan groups. ${ }^{*}<0.05$, $* * p<0.01$, and ${ }^{* *} \mathrm{p}<0.001$ Comparisons according to control group, $++p<0.01$ and $+++p<0.001$ Comparisons according to MTX group. 


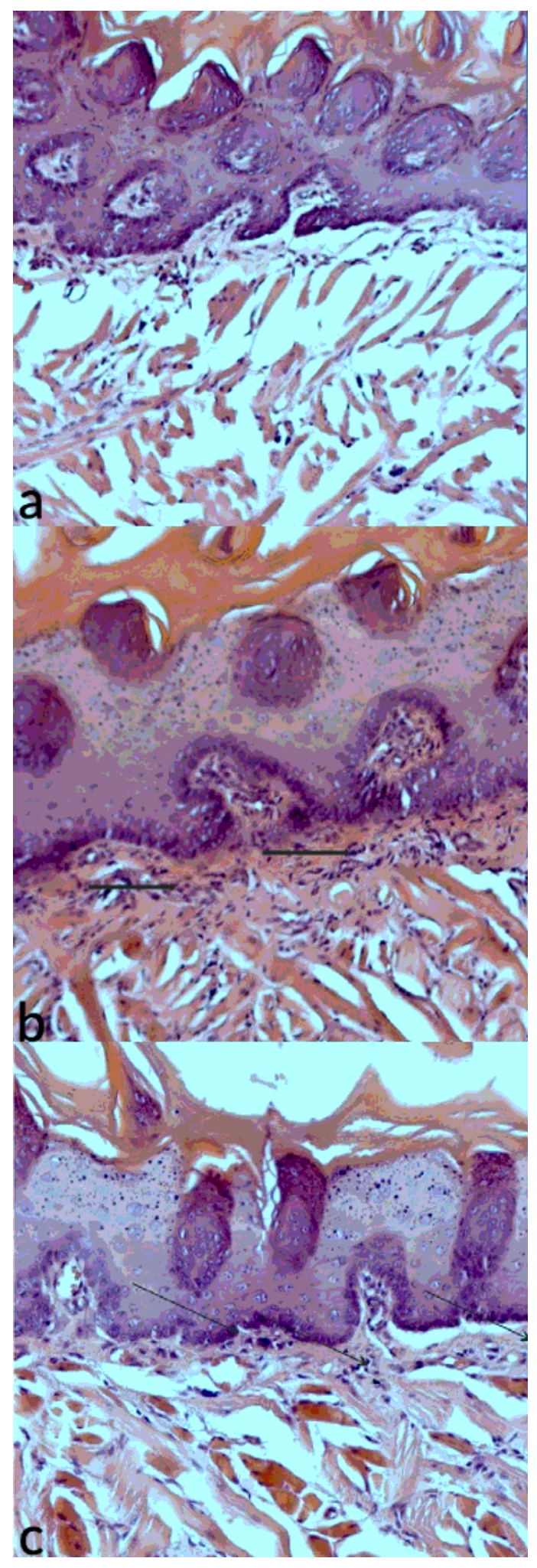

\section{Figure 4}

Histological view of tongue tissue a) Control group b) MTX group C) MTX + Chitosan group, Magnification X200. 


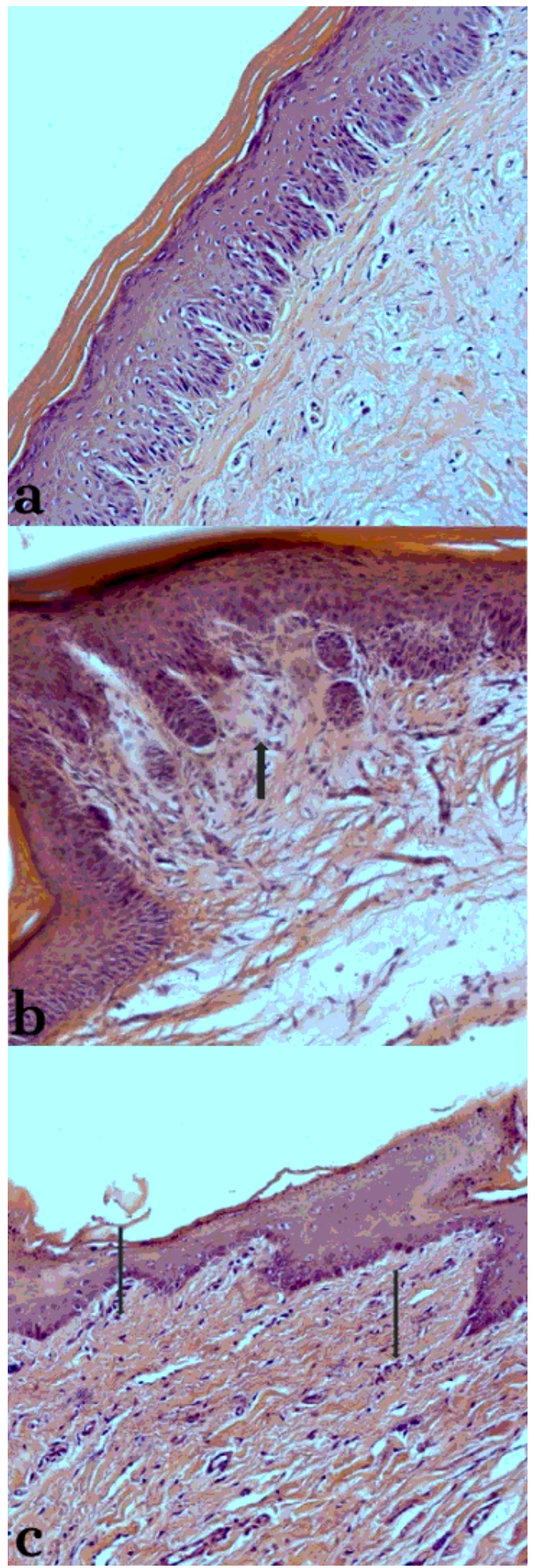

Figure 5

Histological view of palatal tissue a) Control group b) MTX group C) MTX + Chitosan group, Magnification X200. 


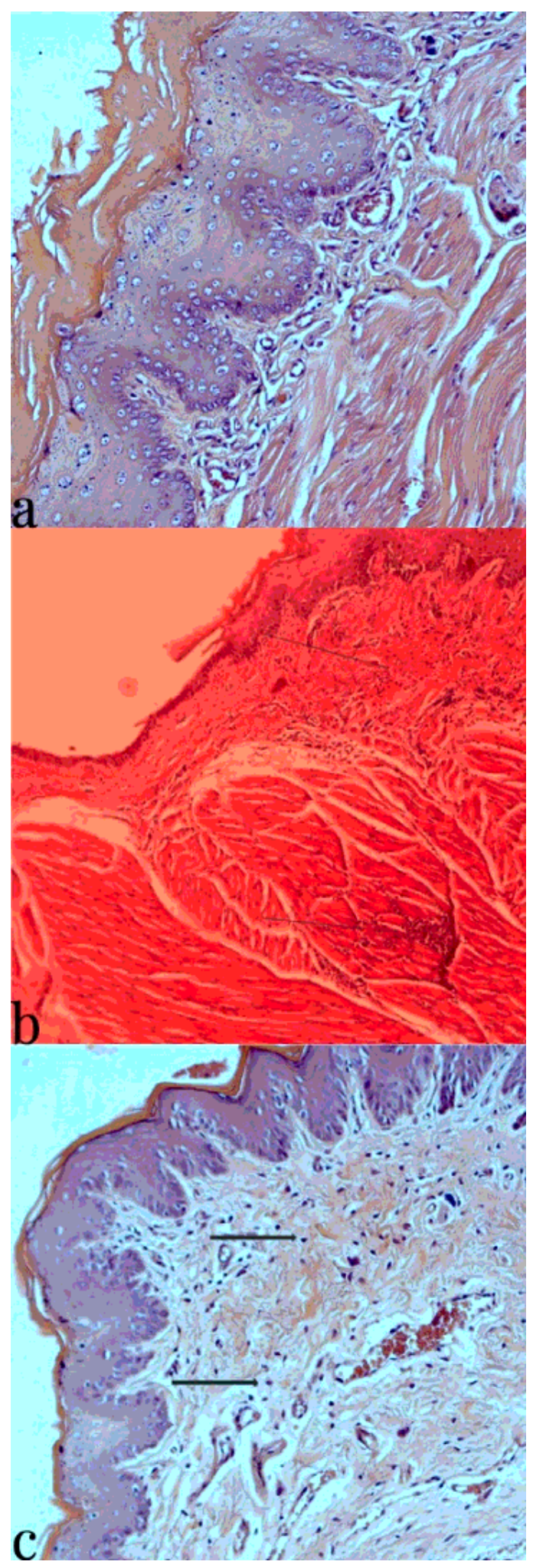

Figure 6

Histological view of buccal tissue a) Control group b) MTX group C) MTX + Chitosan group, Magnification X200. 